\title{
Fabrication of ultra-small GSH-AgNCs with excellent eschar penetration and antibacterial properties for the healing of burn infection wounds
}

\section{Peng Yang}

Nanchang University Second Affiliated Hospital

\section{Kang Yang}

Nanchang University Medical College: Medical College of Nanchang University

Lu Liu

Nanchang University Second Affiliated Hospital

Chen Li

Nanchang University Second Affiliated Hospital

Jun Tao

Nanchang University Second Affiliated Hospital

Fanrong Ai ( $\square$ afr3755875@126.com )

Nanchang University School of Mechanical and Electrical Engineering

\section{Research Article}

Keywords: Silver nanocluster, Burn wound healing, Antibacterial, Fluorescence, Excretion

Posted Date: March 17th, 2021

DOl: https://doi.org/10.21203/rs.3.rs-302056/v1

License: (c) (1) This work is licensed under a Creative Commons Attribution 4.0 International License. Read Full License 


\title{
Fabrication of ultra-small GSH-AgNCs with excellent eschar penetration and antibacterial properties for the healing of burn infection wounds
}

Peng Yang ${ }^{1} \dagger$, Kang Yang $^{2} \dagger, Y u$ liu $^{3}$, Chen $\operatorname{Li}^{1}{ }^{1} \mathrm{Jun} \mathrm{Tao}^{1 *}$ and Fanrong $\mathrm{Ai}^{4,5 *}$

\begin{abstract}
There is evidence of bioburden as a barrier to chronic burn wound healing. Compared to traditional therapy, nanotechnology has availed a revolutionary approach to therapeutic and diagnostic applications in burns. In this article, we developed the glutathione-protected Ag nanoclusters (GSH-AgNCs) to manage burn wound infection. Owing to the specific structure, the GSH-AgNCs emitted strong red fluorescence under UV excitation, quantified via both in vivo and in vitro techniques. The GSH-AgNCs showed a significant inhibition potential on the proliferation of Staphylococcus aureus (S. aureus), Escherichia coli (E. coli), Pseudomonas aeruginosa (P. aeruginosa), and methicillin-resistant staphylococcus aureus (MRSA), hiding under the eschar. Of note, with 2-6nm particle size, GSH-AgNCs are effected in renal excretion, advocating for their biomedical and pharmacological applications.
\end{abstract}

\section{Keywords}

Silver nanocluster, Burn wound healing, Antibacterial, Fluorescence, Excretion 

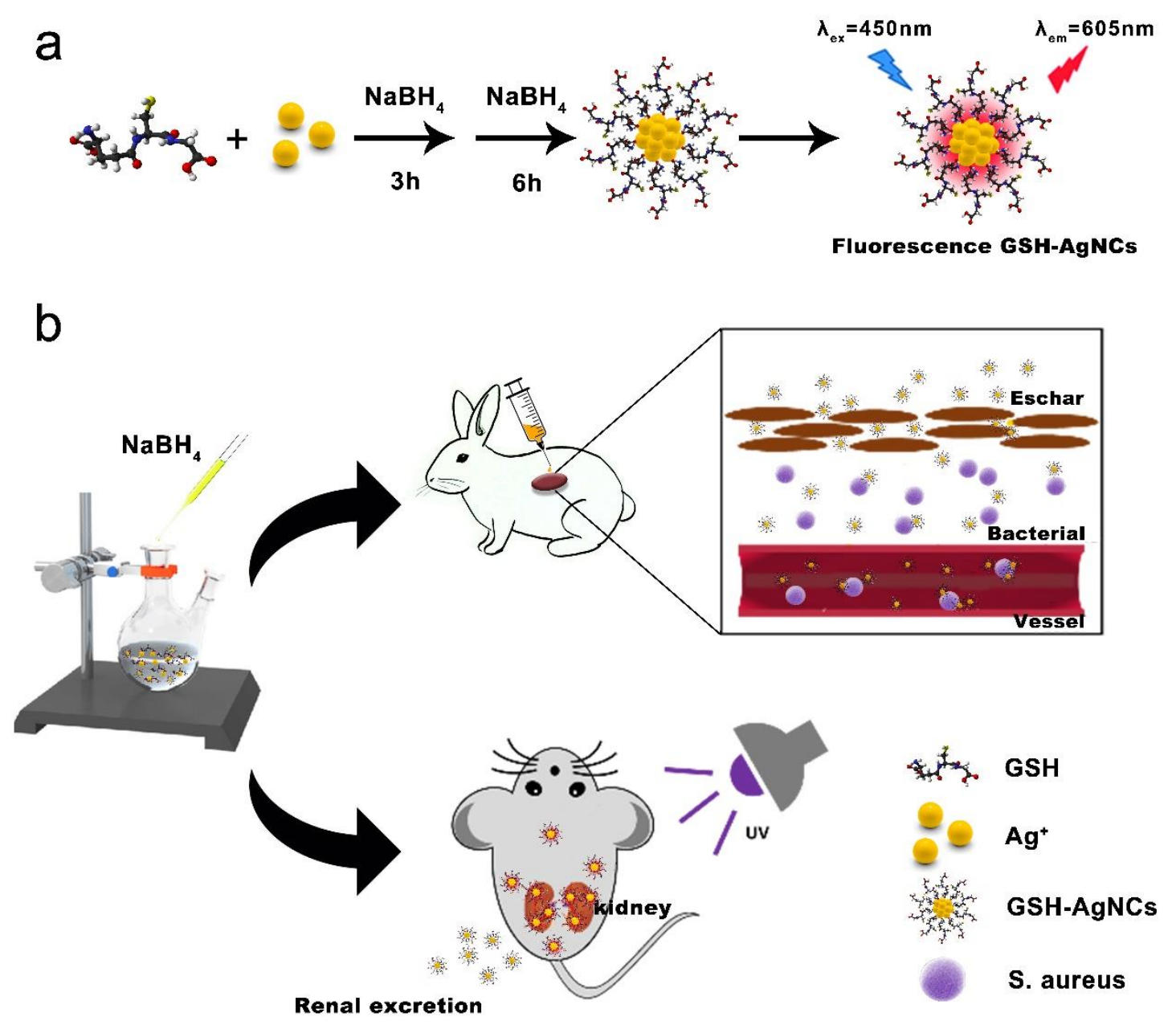

Scheme 1. (a). Schematic diagram of the preparation of glutathione-protected nanocluster silver. (b). They application in the treatment of burns and scab wounds infected by Staphylococcus aureus and in vitro fluorescence monitoring of its renal excretion capacity.

\section{Introduction}

Skin is the largest organ in the body. It is a crucial barrier that blocks the entry of pathogens in vivo. Burn injury-associated impairment of the anatomical structure and function cause wounds ${ }^{[1]}$. Of note, the openness of burn wounds, protein coagulation, and exudation of plasma components create ideal conditions for bacterial growth. Consequently, invasive infection complications caused by sepsis readily occur, particularly when the systemic immune function is compromised ${ }^{[2,3]}$. An estimated 
180,000 burn injury-associated deaths are reported every year, according to the World Health Organization. Sepsis and the accompanying invasive infection remain the primary causes of such burn deaths ${ }^{[4-8]}$. So, antibacterial treatment is essential for burn injury healing.

Although antibiotics are applied as a traditional burn infection therapy ${ }^{[9,10]}$, their long-term use has contributed to a rise in cases of microbial drug resistance, organ damage, allergic reaction, among other adverse side effects ${ }^{[11,12]}$. A series of new approaches have been proposed as escape routes for such complications, including new antimicrobial agents (such as antimicrobial peptides, amino acid-based surfactants, etc.), antimicrobial photo- and ultrasound-therapy, and natural products such as honey ${ }^{[13-17]}$. Among these, nanomedicine as a novel approach has been firmly applied to manage burn infections.

Systemic antibacterial drugs can hardly land on the infected site; thus, topical antimicrobials and dressings are generally employed to enhance wound healing and resist reinfection ${ }^{[18]}$. Full-thickness burn wounds usually form eschars, making it difficult for topical antibacterial agents to act on deep infections ${ }^{[19]}$. Moreover, the excellent penetrating ability of nanoclusters has received immense applications in transdermal drug delivery, brain-targeting, and tumor targeting therapy ${ }^{[20-23]}$. Despite unclear mechanisms underlying tissue barrier permeability, accumulating evidence insinuates that the small size of nanomaterials exerts a pivotal role in tissue penetration. Numerous reports also found that Ag nanoparticles smaller than $30 \mathrm{~nm}$ can penetrate the deepest part of the stratum corneum ${ }^{[24-28]}$. Thus, we hypothesize that the GSH-AgNCs 
(2-6nm in size) designed in this work could demonstrate good permeability.

The widespread application of nanotechnology in medicine is highly promising in the treatment of several diseases. Regrettably, their toxicity remains a potential limiting challenge to developing various nanotechnologies for clinical translation ${ }^{[29]}$. Numerous contributes to the cytotoxicity and genotoxicity of silver nanoclusters; for instance, the first toxicity reaction after in vivo injection with sliver nanoclusters forms the protein corona. Biological response to these coronas is critical for nanotoxicology. Various proteins (i.e., human serum albumin, tubulin, ubiquitin and yeast extract proteins, etc.) are adsorbed onto the surface of sliver nanoclusters ${ }^{[30]}$. Glutathione is a natural tripeptide with a high affinity to metal surfaces. Silver nanoclusters protected by GSH potentially acquire better biocompatibility by blocking the formation of corona ${ }^{[31]}$.

Particle size is an important factor influencing nanocluster distribution. Smallersized nanoparticles are more widely distributed than large-sized ones. In particular, reports show that $10 \mathrm{~nm}$ diameter silver nanoparticles are enriched in several organs (e.g., liver, spleen, kidney, testis, thymus, heart, lung and brain, etc.). Larger nanoparticles are specific to blood, liver, and spleen, implicating that 2-6 $\mathrm{nm}$ diameter silver nanoparticles are more evenly distributed and less enriched in tissues ${ }^{[32-34]}$. In vivo, the nanoparticles continuously induce inflammation and other toxic reactions. Thus, if they do not get cleared, the long-term fate of organ affords is not clear. The renal filtration threshold of nanoparticle size is generally $5.5 \mathrm{~nm}$. However, there is no report on whether larger nanoparticles are metabolized by the kidney, which may be related to its rigidity ${ }^{[33,35-37]}$. 
In most cases, sepsis occurs secondary to severe infection, pneumonia, burn, and major surgery. Therefore, systemic administration must be considered for effective invasive infections prevention and treatment ${ }^{[2,8]}$. Since pharmacokinetics after burn trauma is challenging to predict, real-time therapeutic drug monitoring (TDM) is essential for most antibiotic therapy for burns, which maintains the safe plasma concentration ${ }^{[38,39]}$. The pharmacokinetic models of NPs are considered multicompartment models. However, its distribution and excretion are affected by various factors, posing challenges in predicting the risk of adverse effects using plasma concentration. Herein, inspired by tumor fluorescent markers, we propose a new strategy for adjusting the dosage and enhance the safety of nanomedicine through in vivo examination of nanocluster metabolism ${ }^{[40-42]}$.

This work proposes a new approach to reduce health risks by integrating fluorescent tracer and renal excretion. It is argued that GSH-AgNCs possess an excellent broad-spectrum antibacterial activity, which potentially promotes wound healing. It introduces eschars into the bloodstream to prevent secondary sepsis and invasive infections. The unique fluorescence-exciting properties can grasp the degree of enrichment in the tissue. This justifies the excellent permeability and antibacterial effects of GSH-AgNCs. Nevertheless, excretion greatly improves the antibacterial activity and biosafety of silver nanoparticles.

\section{Materials and methods}

\section{Materials}


Silver nitrate (S116265, Aladdin, China), S-Hexylglutathione (H121370, Aladdin, China), Sodium borohydride (S817796, Macklin, China), Sodium hydroxide (S111509, Aladdin, China), LB Broth medium (HB0128, Hopebio), agar(A8190, Solarbio), Hematoxylin-Eosin/HE Staining Kit (G1120, Solarbio, China). To prepare full cell media, we constituted Dulbecco's Modified Eagle's Medium (DMEM, BI) supplemented with $10 \%$ fetal bovine serum (FBS, BI) and $1 \%$ penicillin-streptomycin. Cell Counting Kit 8 (CCK-8) and Calcein-AM/Propidium Iodide (PI) were purchased from Beyotime Biotechnology.Staphylococcus aureus (S.aureus), Escherichia coli (E.coli), Pseudomonas aeruginosa (P. aeruginosa) and methicillin-resistant staphylococcus aureus (MRSA) were purchased from National Center for Medical Culture Collections (CMCC). Human Skin Fibroblast (HSF) was obtained from The Second Affiliated Hospital of Nanchang University. All animal experiments were approved by the Animal Experiments Ethical Committee of Second Affiliated Hospital of Nanchang University.

\section{Preparation of glutathione-protected Ag nanoclusters}

The material synthesis process was adopted as previously described. Briefly, $125 \mu \mathrm{L}$ of $20 \mathrm{mM}$ silver nitrate and $150 \mu \mathrm{L}$ of $50 \mathrm{mM}$ glutathione were dissolved in $4.85 \mathrm{~mL}$ of water. Afterward, $50 \mu \mathrm{L}$ of $0.1 \mathrm{mM}$ sodium borohydride dissolved in $0.2 \mathrm{mM}$ sodium hydroxide was added for 5 minutes, aged for $3 \mathrm{~h}$. Then, $50 \mu \mathrm{L}$ of boron was added. The sodium hydride solution was stirred for $15 \mathrm{~min}$ and aged for $6 \mathrm{~h}$.

\section{Characterization of GSH-AgNCs}

The structural and size properties of the GSH-AgNCs were examined using 
transmission electron microscopy JEOL 03040701(Kabushiki Kaisha, Japan). We applied the fluorescence spectrometer FS5 (Edinburgh Instruments, UK) to measure the fluorescence spectra. UV-Vis absorbance spectra were recorded with a Cary UV-Vis spectrophotometer.

\section{Cell culture and in vitro cytotoxicity experiment}

The cytotoxicity of GSH-AgNCs was evaluated through direct culturing of HSF cells in high glucose DMEM supplemented with 10\% FBS and 1\% penicillin/streptomycin. This was followed by incubation at $37^{\circ} \mathrm{C}$ in a humidified atmosphere of $5 \% \mathrm{CO} 2 / 95 \%$ air. For long-term toxicity evaluation, we assessed cell growth. Briefly, $1 \times 104$ cells were seeded on a $24-$ well plate, and then treated with $125 \mu \mathrm{M}, 250 \mu \mathrm{M}$ GSH-AgNCs for 1, 3, or 7 days. Cell growth was evaluated under the microscope. Following a 5-day incubation, a life/death analysis kit was used to assess the killing effect of GSH-AgNCs on HSF cells. The dyes in the kit include calcein (AM) and propidium iodide (PI, which stains live cells (green) and dead cells (red), respectively. A fluorescence microscope was used to record cell viability.

Measurement of the concentration-toxicity relationships of GSH-AgNCs in different cell lines was taken using CCK-8 assay according to the manufacturer's protocol. Here, HSF cells were seeded onto 96 -well plates overnight $(1 \times 103$ cells per well) and subsequently treated with varying concentrations of GSH-AgNCs $(0,7.8125$, $15.625,31.25,62.5,125,250,500$, or $1000 \mu \mathrm{M})$, in triplicate for each experimental group. For the negative control group, we used a concentration of GSH-AgNCs at 0 $\mathrm{mM}$, whereas medium without cells served as the blank control. Then, $10 \mu \mathrm{L} \mathrm{CCK}-8$ 
solution was added into each well at 1, 3, and 7days post-seeding. Each group was cultured for a further 2 hours. The optical density values at $450 \mathrm{~nm}$ were measured using a microplate reader. An estimate of cell survival rate (\%) of each cell lines treated with various concentrations of GSH-AgNCs was measured as follows: [(OD value of negative control - OD value of experimental group)/ (OD value of negative control OD value of blank control).

\section{In vitro antibacterial activity}

The spread plate method was adopted to evaluate in vitro antibacterial activity. Two Gram-negative bacterial strains, P. aeruginosa and E. coli, and two Gram-positive bacterial strains, S. aureus and MRSA, were used for this evaluation. We suspended GSH-AgNCs in LB broth medium at the concentrations of $0,15.6,62.5,250$, or 1000 $\mathrm{mM}$. Then, a speed vortex mixer was used to produce dispersions. The abovementioned microorganisms were added to the Ag nanoclusters suspensions. The positive control comprised the LB broth medium with the microorganism and without GSH-AgNCs, whereas the negative control comprised the LB broth without the microorganism. After $8 \mathrm{~h}$ culture at $37^{\circ} \mathrm{C}$, all the tested suspensions were diluted $(1 \times 105$ fold $)$ and inoculated onto nutrient agar plates. A count of CFUs on agar plates was taken after $12 \mathrm{~h}$ of culture. Antibacterial efficacy (\%) of GSH-AgNCs at each concentration was estimated as: [(CFU count of negative control-CFU count of experimental group)/CFU count of negative control] $\times 100 \%$.

Using Oxford cup, we further evaluated the transdermal antimicrobial efficiency of the GSH-AgNCs. Obtained eschar skin from rabbit infected burn wound. Afterward, 
the eschar was placed on plates previously inoculated with the pathogen. Different concentrations of nanoclusters dispersions were added to each Oxford cup and placed on the above artificial eschar. After $24 \mathrm{~h}$-incubation at $37^{\circ} \mathrm{C}$, images of the bacterial zone of inhibition were taken using camera.

\section{Fluorescence imaging in vivo}

The fluorescence of the GSH-AgNCs was assessed using In Vivo Imaging System LB983 (BERTHOLD, USA). In vivo imaging was performed immediately after caudal vein injection. All the experimental Kunming mice were purchased from Jiangxi University of Chinese Traditional Medicine. The control mice were injected with the same amount of PBS. Whole-body images of GSH-AgNCs injected mice were acquired and analyzed at different time points $(0.5,1,2,3,4,6,8,12$ and 24 hours $)$.

\section{Systemic toxicity}

Although the GSH-AgNCs could penetrate eschars into the bloodstream to prevent and treat systemic infection, it potentially causes systemic toxicity. Herein, the histopathology of organs was investigated for systemic toxicity evaluation. Briefly, seven days following a single intravenous dose of GSH-AgNCs suspension, mouse eyeballs were extracted to collect blood. Using Automated Clinical Chemistry Analyzer (AU400, Olympus), we tested the total bilirubin (ALST), alanine aminotransferase (ALT), aspartate aminotransferase (AST), alkaline phosphatase (ALP), urea nitrogen (BUN), and serum creatinine (SCR) to evaluate the hepatic and renal damage after GSH-AgNCs suspension inject.

The microstructure destruction of organs was evaluated via HE staining. Briefly, 
organ tissues from harvested heart, liver, spleen, lung, and kidney from the above mice were fixed with $10 \%$ buffered formalin for $24 \mathrm{~h}$. Tissues were then paraffin-embedded, sectioned, and subjected to HE staining following the manufacturer's protocol. Histopathological changes were examined under a light microscope.

\section{Burn infection model}

New Zealand white rabbits were purchased from the Institute of Animal Science of Nanchang University and randomly categorized into two groups. We adopted the following steps to cause burns and infections. Briefly, after removing the back hair of the rabbits, they anesthetized through the marginal ear vein of chloral hydrate at a dose of $250 \mathrm{mg} / \mathrm{kg}$. Borrowed from previous reports, a full-thickness burn wound was made on the dorsal skin using copper coin $\left(11 \mathrm{~mm}, 92^{\circ} \mathrm{C}, 25 \mathrm{~s}\right)$. The Staphylococcus aureus suspension $(200 \mu \mathrm{L}, 1 \times 105$ cells $\mathrm{mM}-1)$ was injected subcutaneously into the burn. The GSH-AgNCs suspension was dropped on the eschar after the wound had scabs. After that, the wound was covered with gauze and fixed with an elastic bandage. Images of the wound at the planned time points $(0,3,7$, and 10 days $)$ were taken. The wound was cut and opened with scissors to check for infection. The surrounding gauze was removed. The antibacterial effect and tissue inflammation were evaluated using the hematoxylin-eosin staining kit.

\section{Statistical analysis}

Statistical computations were performed using one-way ANOVA between multiple groups. Data were expressed as mean \pm standard deviation (SD). All statistical computations were performed using GraphPad Prism 8.0.1. The significance level was 
set as $\mathrm{p}<0.05$.

\section{Results and Discussion}

\section{Characterization of GSH-AgNCs}

The prepared GSH-AgNCs were characterized via TEM, UV-Vis absorption, and fluorescence spectra (Figure 1). We have presented a typical TEM image of GSHAgNCs nanoparticles in Figure 1a. GSH @ Ag NCs were spherical, well dispersed, and uniform. The particle size analysis of the nanoparticles confirmed the smaller size of GSH-AgNC, about $2.5 \mathrm{~nm}$. The purified GSH-AgNCs was brown in solution and demonstrated a pronounced absorption peak at 450nm (Figure 1b). The changes in product fluorescence intensity measured under excitation light with wavelengths of 400 , $410,420,430,440$, and $450 \mathrm{~nm}$ are shown in Figure 1c. The emission spectrum was found at $605 \mathrm{~nm}$; at this point, we noted the strongest fluorescence intensity. The excitation and emission spectra of GSH-AgNC, respectively, are presented in Figure 1d. Notably, the excitation spectrum monitored at $605 \mathrm{~nm}$ produced a broad excitation band from 400 to $500 \mathrm{~nm}$ (black line). Further, GSH-AgNCs showed a sharp excitation peak (red line) at 450nm. The synthesized cluster-shaped silver nanoparticles emitted red fluorescence visible to the naked eye under ultraviolet light, which could be explained by the formation of fluorescent AgNCs.

Based on previous findings, the characteristic absorption peaks at around 380-500 $\mathrm{nm}$ as found in GSH-AgNCs are for larger Ag nanoparticles [31,43]. This could be attributed to the interparticle assembly of uncapped silver nanoclusters. 

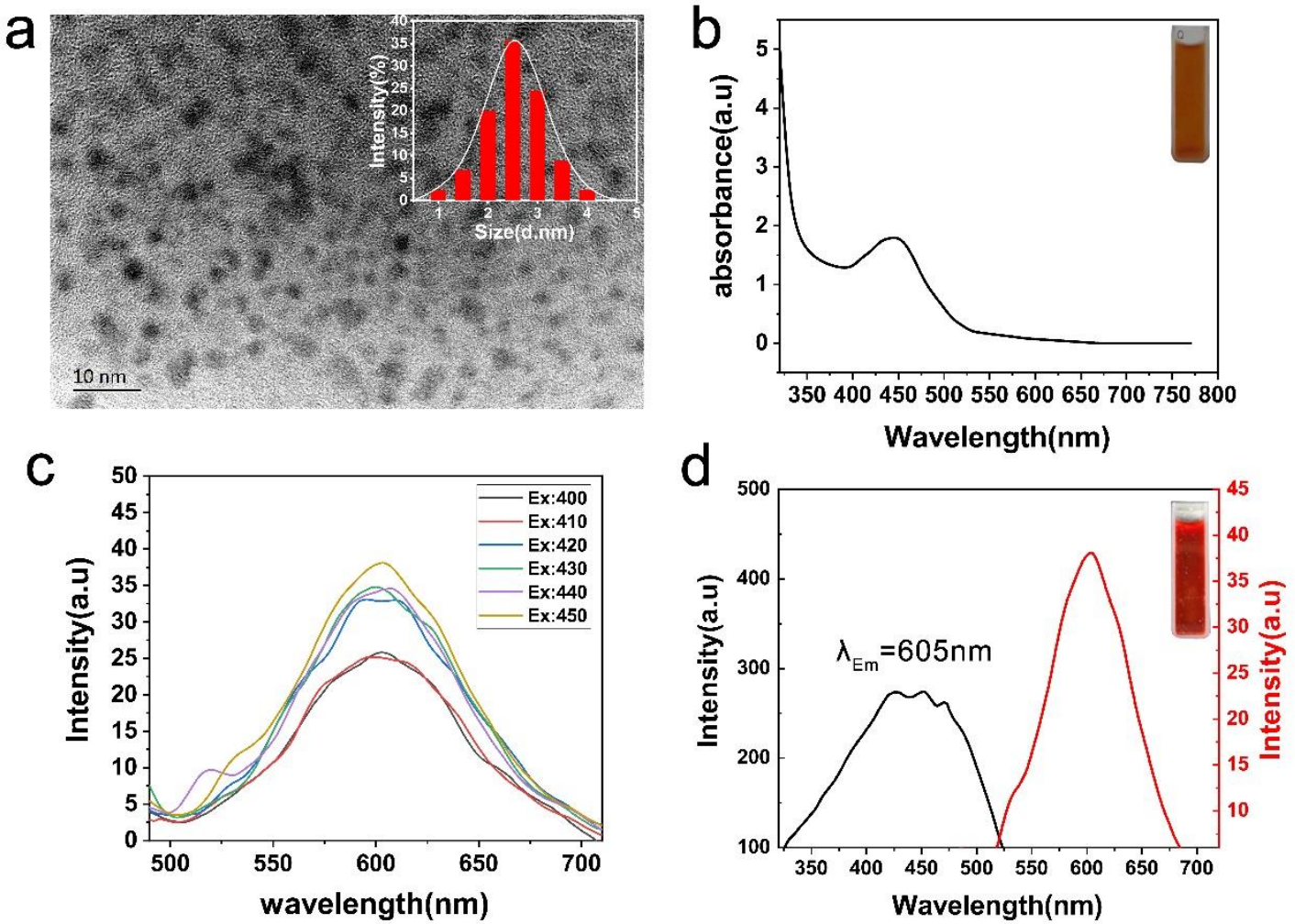

Figure 1. (a). TEM image of the GSH-AgNCs. Scale bar: $10 \mathrm{~nm}$. Illustration: Particle size distribution. (b). UV-vis spectroscopy of GSH-AgNCs, the inset shows the photo of GSH-AgNCs under visible light irradiation. (c). Changes in the fluorescence intensity of GSH-AgNCs measured under the action of different wavelengths of excitation light. (d). Excitation spectrum (black lines) and emission spectrum (red lines) of GSH-AgNC at $605 \mathrm{~nm}$ and $450 \mathrm{~nm}$. The inset shows a photo of GSH-AgNCs emitting red fluorescence under UV irradiation.

\section{Proliferation and in vitro viability}

HSF cells gradually proliferated within 7 days and covered the entire culture dish over time (Figure 2a). There was almost no difference in the average cell number of the control group without cluster silver. Notably, cells incubated with different concentrations of GSH-AgNCs were all alive ( Figure 2b). After 5 days of culture, the live cells (green) and propidium iodide (PI) were paired with calcein-AM (Cal-AM). After staining the dead cells (red), both the $125 \mu \mathrm{M}$ and $250 \mu \mathrm{M}$ GSH-AgNCs 
experimental groups survived, slightly less than the average cell number of the control group. It was suggested that GSH potentially reduced the toxicity of silver ions, thereby promoting the proliferation of HSF cells.

Furthermore, CCK-8 analysis was undertaken to quantitatively analyze the toxicity of GSH-Ag NCs to LSF cell survival. We used different concentrations of GSH-Ag NCs and HSF for 1, 3, and 7 days of co-cultivation to validate the results of the cck8 experiment (Figure 2c). Of note, the toxicity of GSH-Ag NCs to cells was dose-dependent. Compared to the control group, the survival rate of cells below $250 \mu \mathrm{M}$ was not affected.

a
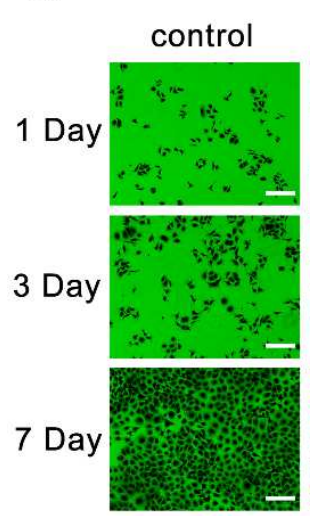

C

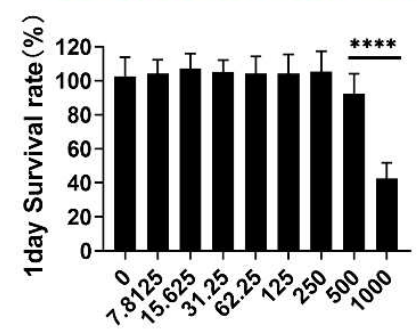

b
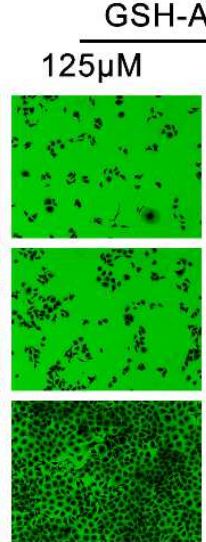

$250 \mu \mathrm{M}$
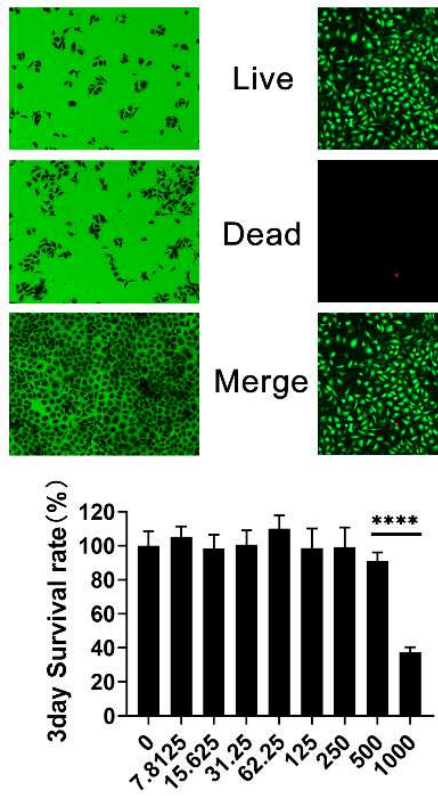

b
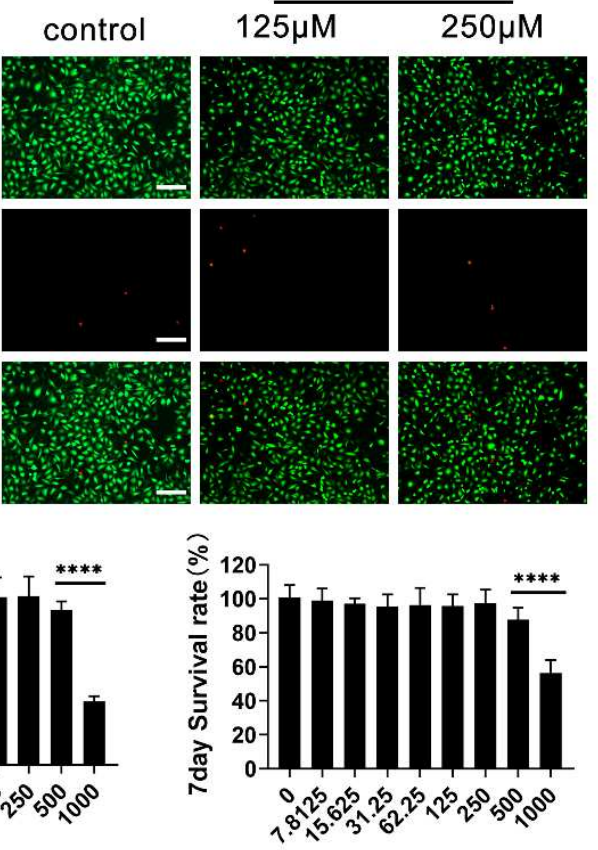

Figure 2. (a). Fluorescence inverted microscopy of the images of $125 \mu \mathrm{M}, 250 \mu \mathrm{M}$ GSH-AgNC, and HSF incubated together for 1, 3, and 7 days. Scale bar: $200 \mu \mathrm{m}$. (b). Live/dead fluorescent staining images of HSF cells incubated with different GSH-AgNCs after 5 days. Live cells are labeled with green fluorescence, while dead cells are labeled with red fluorescence. Scale bar: $200 \mu \mathrm{m}$. (c) The results of CCK-8 after co-culturing HSF cells with different concentrations of GSH-AgNCs for 1, 3, and 7 days. $\left({ }^{*} \mathrm{P}<0.05\right)$. 
Collectively, GSH-AgNCs concentration lower than $250 \mu \mathrm{M}$ demonstrated satisfactory biocompatibility, which is beneficial to the growth and proliferation of HSF cells, thus, has potential biomedical applications.

\section{In vivo biocompatibility}

Silver nanoclusters display intense colors due to the collective oscillation of conduction electrons as they interact with light. Owing to their fluorescent properties, metal nanocluster materials received wide application in tumor imaging, microRNA detection, and DNA base detection [49-51]. Here, the synthesized silver nanoparticles had been tested for their in vitro fluorescence capabilities previously. The real-time fluorescence in vivo performance of GSH-AgNCs was investigated on Kunming mice (Figure 3a). Similarly, it demonstrated good fluorescence imaging capabilities in vivo, thus can be applied as a dynamic biological probe. We observed the in vivo fluorescence signal after injection (Figure 3a). The signal of nanoparticles was further attenuated over time, which was not due to the fluorescence quenching of GSH-AgNCs but because they were excreted. After $24 \mathrm{~h}$ post-injection, most GSH-AgNCs had been cleared out. The accumulation site of residual nanoclusters confirmed that GSH-AgNCs is renal excretion type, consistent with the theoretical pharmacokinetics of 2-6nm diameter nanocluster. Results of major liver and kidney function tests (including ALT, AST, ALP, BUN, SCR, TBIL) after intravenous injection of GSH-Ag NCs suspension are presented in Figure 3b. Compared to the control group, the experimental group had no significant difference in various indicators on the 7th day. This implied that GSHAgNCs nanoparticles did not influence the normal liver and kidney basic physiological 
functions of the mice post injection. H\&E-stained tissue sections from the main organs (heart, liver, spleen, lung, and kidney) of km mice 7 days after a single intravenous injection of GSH-Ag NCs suspension are depicted in Figure 3c. Mice in the control group received $200 \mu \mathrm{L}$ of normal saline intravenously but showed no obvious pathological changes.

a
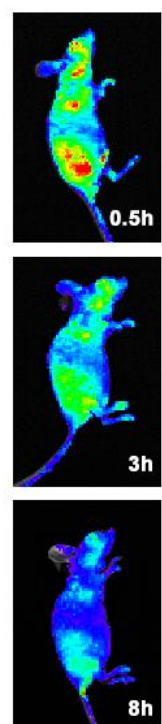
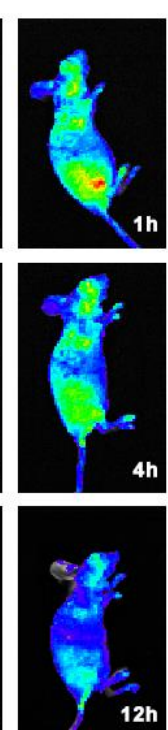
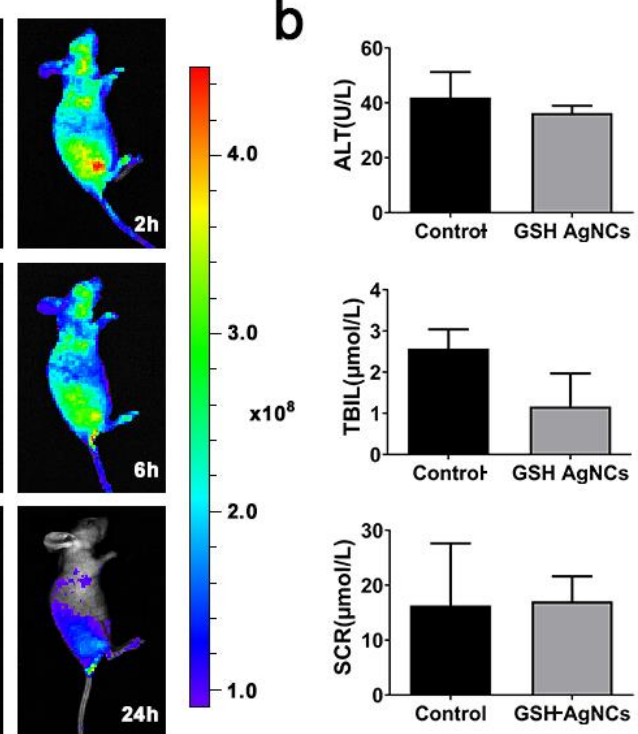
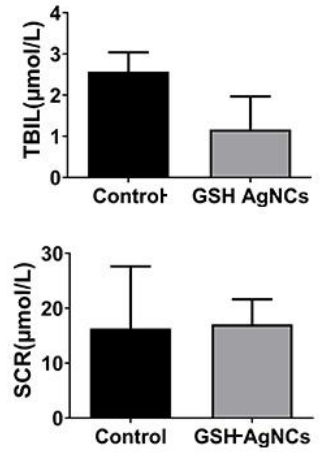
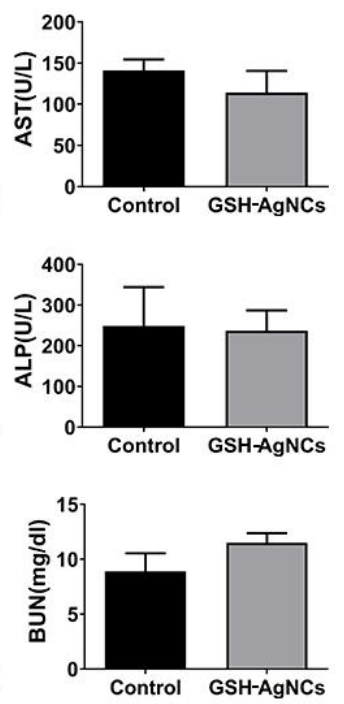

C

Heart

liver Kidney

Spleen

Lung

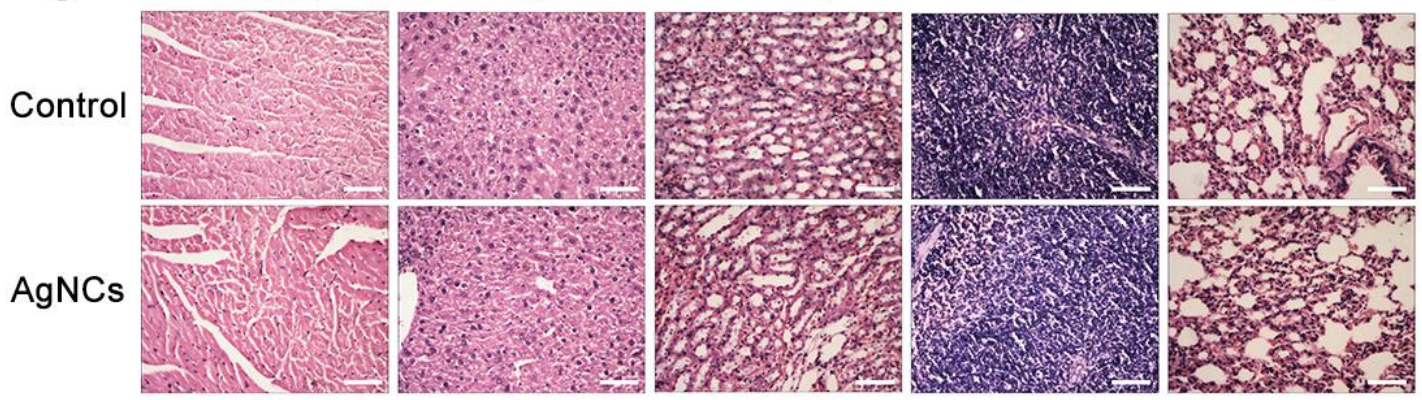

Figure 3. (a). Fluorescence images at different times point after injection of GSH-AgNCs. (b). Evaluation of the liver function (ALT, AST, TBIL, ALP)) and kidney function of km mice injected with GSH @ Ag NCs (BUN, SCR); mice in the control group received an intravenous injection of $200 \mu \mathrm{L}$ of normal saline. (c). Seven days after a single intravenous injection of GSH-Ag NCs suspension, H\&E-stained tissue (heart, liver, spleen, lung, and kidney) sections were taken from km mice. Scale bar: $200 \mu \mathrm{m} .(\mathrm{n}=3)$.

\section{In vitro antibacterial activity}


The microbial load reduces burn wound contraction, and eventually, death may occur. Silver and associated preparations have been applied as antimicrobials for thousands of years ${ }^{[46]}$. Though the mechanism of antibacterial activity of AgNCs remains elusive, theories are hypothesizing that they (I) react with thiol moieties of enzymes and proteins, (II) produces free radicals, (III) causing membrane structure damage ${ }^{[47,48]}$. It is worth mentioning that the specific surface area of sliver nanoclusters exerts a crucial role in the adsorption and destruction of proteins. Herein, the antibacterial property of GSHAgNCs was quantitatively assessed against both Gram-positive and Gram-negative bacteria using CFU count. The data are presented in Figure 4a, 4c. For a similar antibacterial effect, the Gram-negative species required a larger GSH-AgNCs exposure concentration than the Gram-positive species. Excellent antibacterial effect against both Gram-negative and Gram-positive bacteria was recorded at concentrations as low as $62.5 \mu \mathrm{M}$

Eschar formation in the infected area of burns is an important factor in antibacterial treatment failure ${ }^{[7]}$. Referring to the upper schematic diagram (Figure 4b), adding different concentrations of GSH-AgNCs to each Oxford cup enhance the penetration of nanoclusters of small particles into skin eschar at the bottom. Eventually, the Staphylococcus aureus pre-inoculated on the plate die.

The image of the bacterial inhibition zone taken after adding cluster silver to the Oxford cup followed by $24 \mathrm{hr}$ incubation at $37^{\circ} \mathrm{C}$ is shown in Figure $4 \mathrm{~b}$. GSH-Ag NCs penetrated the eschar, exerting an antibacterial effect. The variation in the area of the inhibition zone with the concentration of GSH-AgNCs shows that it can penetrate the 
eschar, killing the bacteria in the wound and blood at the bottom of the eschar. We speculate that this may be due to the small size of clustered silver nanoparticles, and the naturally occurring small peptide GSH can act as a protective shell, so GSH-AgNCs show excellent permeability in the eschar tissue.

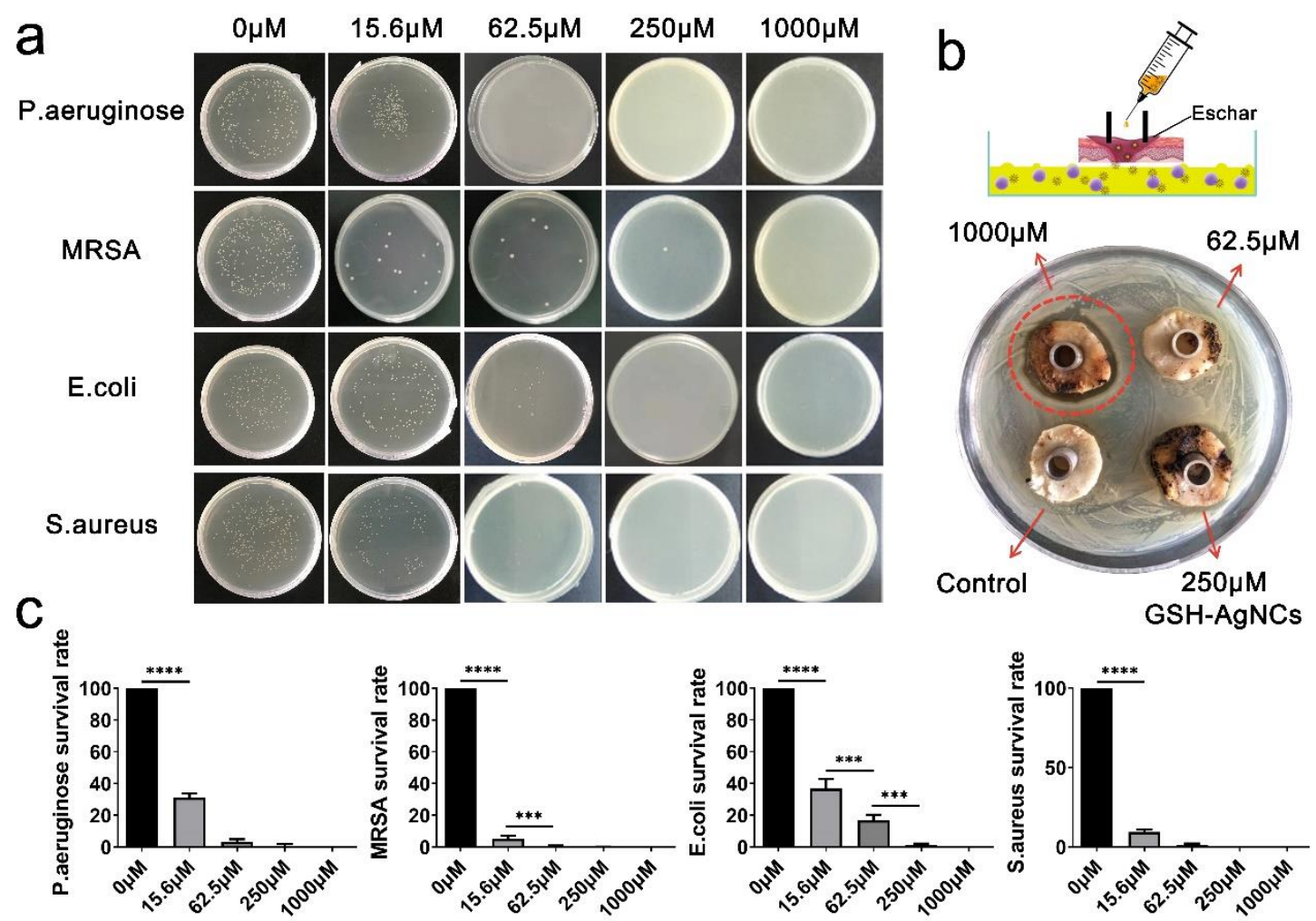

Figure 4. (a). Antibacterial efficiency of GSH-AgNCs against different bacteria using the Coated plate method. (b). The pictures and simple schematic diagrams of GSH-AgNCs permeating the skin eschar to form a zone of inhibition. (c). Histogram representation of the bacterial survival rate results.

\section{Treatment of infected burn wounds}

After introducing a burn on the shaved back of New Zealand white rabbit using a preheated brass block $\left(92^{\circ} \mathrm{C}\right)$ for 25 seconds, Staphylococcus aureus was injected subcutaneously into the wound. Two days later, the wound crusted. An abscess occurred after cutting the wound with scissors. This meant that a closed full-thickness burn wound infection model was successfully established. 
As shown in the schematic diagram of Figure 5a,to evaluate the effects of the GSH-AgNCs on the closing burn wound in vivo, a full-thickness burn wound infection model was used in the rabbit. The subsequent wound repair processes of the treated burn infection wounds were tracked over 10 days. The wounds were either dressed with PBS (blank) or treated with GSH-AgNCs. Significant differences in wound healing were examined during the entire process. The visual observations of burn wounds treated with GSH-AgNCs at different post-operation time points are presented in Figure 5b. Notably, wounds treated with GSH-AgNCs showed a significantly higher wound healing rate than blank groups tested. Such wounds appeared to be fully recovered at day 10 . On the contrary, we reported a significant delay in the closure of wounds in the blank group. Pus was discharged from the wounds, suggesting the development of severe skin infection. These observations were validated through HE staining (Figure 5c). The GSH-AgNCs treated wounds had more capillaries and fibroblasts. After 10 days, no obvious inflammation occurred, and the healing outcome was better. Nevertheless, there were several neutrophils and necrotic nuclei under the infection wound in the control groups. Taken together, significantly inhibited inflammation, less necrotic tissue, more regenerated skin appendages, and well-wound closure are better healing outcomes in GSH-AgNCs treated burn infection wounds. 


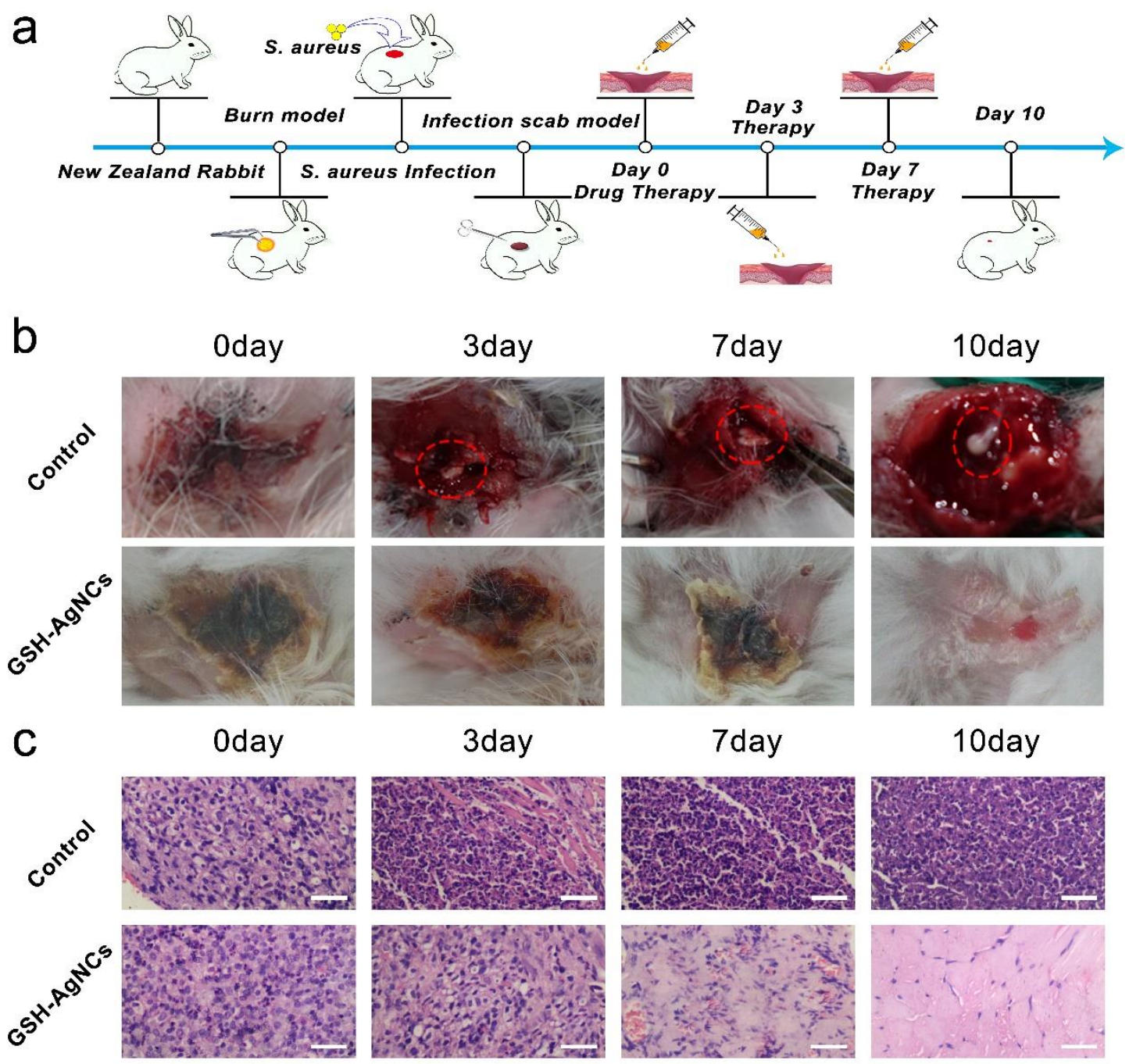

Figure 5. (a). Schematic diagram of the closed full-thickness burn wound infection modeling and GSH-AgNCs treatment process. (b). Wound healing efficacy of GSH-AgNCs in a burning infection wound model in rabbits; red circles indicate pus spots. (c). H\&E staining of the sampled wound tissues after 10 days post-treatment. Scale bar: $200 \mu \mathrm{m}$.

\section{Conclusions}

This study developed a silver nanocluster through glutathione capping, functioning as an antibacterial drug with promising potential for eschar penetration, fluorescent tracing, and renal excretion for burn infection wound healing. Of note, small particle size and surface chemistry offer GSH-AgNCs an excellent permeability; therefore, it 
kills the bacteria under the eschar and prevents secondary sepsis upon entry into the systemic circulation. In vitro and in vivo toxicity analyses show that GSH-AgNCs are less toxic; they undergo renal excretion and get cleared within 24 hours. Also, they maintain fluorescence excitation with a certain intensity in the body for a given time, thus can be adopted as a fluorescent quantitative method to assess the distribution of nanocluster in vivo and regulate the amount of dose to avoid accumulated drug toxicity event. GSH-AgNCs treated wounds demonstrate accelerated wound healing evidenced by faster wound closure, Infection suppression and inflammation inhibition compares to untreated wounds after 10 days. However, future work should focus on the toxic dose effect of GSH-AgNCs on different organs, and establish a model for evaluating in vivo fluorescence intensity and the number of GSH-AgNCs. This would pass the reference for the transdermal treatment system.

\section{Acknowledgements}

This work was supported by the National Natural Science Foundation of China (No.31960207 to Fanrong Ai); China Postdoctoral Science Foundation (No. 2017M610402 to Fanrong Ai); Postdoctoral Science Foundation of Jiangxi Province (No. 2017KY06 to Fanrong Ai); The Science and Technology Project of Jiangxi Education Department (No. GJJ180012 to Jun Tao) ;Key Research and Development Program of Jiangxi Province (No. 20181BBG70026 to Chen Li).

\section{Availability of data and materials}


All the generated or analyzed data during this study are included in this manuscript.

\section{Ethics approval}

The animal study was reviewed and approved by Ethical Committee of Laboratory Animal Science Department, Nanchang University.

\section{Competing interests}

The authors declare that the research was conducted in the absence of any commercial or financial relationships that could be construed as a potential conflict of interest.

\section{Authors' contributions}

PY and KY contributed to conception and design, data acquisition, analysis, interpretation, and drafting of the manuscript. YL and CL contributed to data acquisition and drafting of the manuscript. JT contributed to interpretation and critical revision of the manuscript. FA and JT contributed to conception and design, interpretation, and critical revision of the manuscript. All authors gave final approval and agreed to be accountable for all aspects of the work.

\section{Authors' information}

${ }^{1}$ Department of Orthopaedics, The Second Affiliated Hospital of Nanchang University, Nanchang, PR China.E-mail: 2431835455@qq.com.² Rehabilitation Department, The 
Second Affiliated Hospital of Nanchang University, Nanchang, PR China. ${ }^{3}$

Rehabilitation Department, The Second Affiliated Hospital of Nanchang University, Nanchang, PR China. ${ }^{4}$ School of Mechatronics Engineering, Nanchang University, Nanchang 330031, PR China E-mail: afr3755875@126.com. ${ }^{5}$ Nanchang Municipal Key Laboratory of 3D Bioprinting Technology and Equipment, Nanchang University, Nanchang, Jiangxi, 330031, China. $\uparrow$ These authors contributed equally to this work.* Address correspondence to Fanrong Ai ( E-mail:afr3755875@126.com ) or Jun Tao (Email: 2431835455@qq.com).

\section{References}

[1]. Hänel KH, Cornelissen C, Lüscher B, Baron JM. Cytokines and the skin barrier. Int J Mol Sci. 2013 Mar 26;14(4):6720-45.

[2]. Barajas-Nava LA, López-Alcalde J, Roqué i Figuls M, Solà I, Bonfill Cosp X. Antibiotic prophylaxis for preventing burn wound infection. Cochrane Database Syst Rev. 2013 Jun 6;(6):CD008738.

[3]. Lachiewicz AM, Hauck CG, Weber DJ, Cairns BA, van Duin D. Bacterial Infections After Burn Injuries: Impact of Multidrug Resistance. Clin Infect Dis. 2017 Nov 29;65(12):2130-2136.

[4]. Smolle C, Cambiaso-Daniel J, Forbes AA, Wurzer P, Hundeshagen G, Branski LK, Huss F, Kamolz LP. Recent trends in burn epidemiology worldwide: A systematic review. Burns. 2017 Mar;43(2):249-257.

[5]. GBD 2017 Causes of Death Collaborators. Global, regional, and national age-sex-specific mortality for 282 causes of death in 195 countries and territories, 1980-2017: a systematic analysis for the Global Burden of Disease Study 2017. Lancet. 2018 Nov 10;392(10159):1736-1788.

[6]. Gallaher JR, Purcell LN, Banda W, Charles A. The effect of traditional healer intervention prior to allopathic care on pediatric burn mortality in Malawi. Burns. 2020 Dec;46(8):1952-1957.

[7]. Udy AA, Roberts JA, Lipman J, Blot S. The effects of major burn related pathophysiological 
changes on the pharmacokinetics and pharmacodynamics of drug use: An appraisal utilizing antibiotics. Adv Drug Deliv Rev. 2018 Jan 1;123:65-74.

[8]. Norbury W, Herndon DN, Tanksley J, Jeschke MG, Finnerty CC. Infection in Burns. Surg Infect (Larchmt). 2016 Apr;17(2):250-5.

[9]. Rowan MP, Cancio LC, Elster EA, Burmeister DM, Rose LF, Natesan S, Chan RK, Christy RJ, Chung KK. Burn wound healing and treatment: review and advancements. Crit Care. 2015 Jun 12;19:243.

[10]. Lee KC, Joory K, Moiemen NS. History of burns: The past, present and the future. Burns Trauma. 2014 Oct 25;2(4):169-80.

[11]. Thornhill MH, Dayer M, Lockhart PB, McGurk M, Shanson D, Prendergast B, Chambers JB. A change in the NICE guidelines on antibiotic prophylaxis. Br Dent J. 2016 Aug 12;221(3):112-4. [12]. Ventola CL. The antibiotic resistance crisis: part 1: causes and threats. P T. 2015 Apr;40(4):277-83.

[13].Dai T, Gupta A, Huang YY, Yin R, Murray CK, Vrahas MS, Sherwood ME, Tegos GP, Hamblin MR. Blue light rescues mice from potentially fatal Pseudomonas aeruginosa burn infection: efficacy, safety, and mechanism of action. Antimicrob Agents Chemother. 2013 Mar;57(3):1238-45.

[14].Song A, Rane AA, Christman KL. Antibacterial and cell-adhesive polypeptide and poly(ethylene glycol) hydrogel as a potential scaffold for wound healing. Acta Biomater. 2012 Jan;8(1):41-50.

[15]. Shi Q, Luo X, Huang Z, Midgley AC, Wang B, Liu R, Zhi D, Wei T, Zhou X, Qiao M, Zhang J, Kong D, Wang K. Cobalt-mediated multi-functional dressings promote bacteria-infected wound healing. Acta Biomater. 2019 Mar 1;86:465-479.

[16].Han L, Li P, Tang P, Wang X, Zhou T, Wang K , Ren F, Guo T , Lu X . Mussel-inspired cryogels for promoting wound regeneration through photobiostimulation, modulating inflammatory responses and suppressing bacterial invasion. Nanoscale. 2019 Aug 29;11(34):15846-15861.

[17]. Mandal MD, Mandal S. Honey: its medicinal property and antibacterial activity. Asian Pac J Trop Biomed. 2011 Apr;1(2):154-60.

[18]. Mofazzal Jahromi MA, Sahandi Zangabad P, Moosavi Basri SM, Sahandi Zangabad K, 
Ghamarypour A, Aref AR, Karimi M, Hamblin MR. Nanomedicine and advanced technologies for burns: Preventing infection and facilitating wound healing. Adv Drug Deliv Rev. 2018 Jan 1;123:3364.

[19]. Kalita S, Devi B, Kandimalla R, Sharma KK, Sharma A, Kalita K, Kataki AC, Kotoky J.

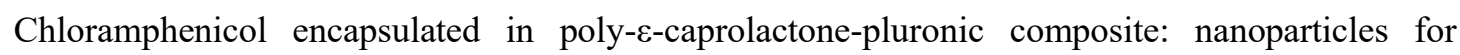
treatment of MRSA-infected burn wounds. Int J Nanomedicine. 2015 Apr 15;10:2971-84.

[20].Wu VM, Huynh E, Tang S, Uskoković V. Brain and bone cancer targeting by a ferrofluid composed of superparamagnetic iron-oxide/silica/carbon nanoparticles (earthicles). Acta Biomater. 2019 Apr 1;88:422-447.

[21].Zadeh B S M, Barati M H H N, Fakher R . Development of Solid lipid nanoparticles as Eschar delivery system for Nitrofurazone Using Taguchi Design Approach[J]. International Journal of Research in Pharmaceutical Sciences, 2010(4):466-472.

[22]. Wang Q, Zou C, Wang L, Gao X, Wu J, Tan S, Wu G. Doxorubicin and adjudin co-loaded pHsensitive nanoparticles for the treatment of drug-resistant cancer. Acta Biomater. 2019 Aug;94:469481.

[23]. Wiraja C, Zhu Y, Lio DCS, Yeo DC, Xie M, Fang W, Li Q, Zheng M, Van Steensel M, Wang L, Fan C, Xu C. Framework nucleic acids as programmable carrier for transdermal drug delivery. Nat Commun. 2019 Mar 8;10(1):1147.

[24].Labouta HI, Schneider M. Interaction of inorganic nanoparticles with the skin barrier: current status and critical review. Nanomedicine. 2013 Jan;9(1):39-54.

[25]. Barua S, Mitragotri S. Challenges associated with Penetration of Nanoparticles across Cell and Tissue Barriers: A Review of Current Status and Future Prospects. Nano Today. 2014 Apr $1 ; 9(2): 223-243$.

[26].Larese FF, D'Agostin F, Crosera M, Adami G, Renzi N, Bovenzi M, Maina G. Human skin penetration of silver nanoparticles through intact and damaged skin. Toxicology. 2009 Jan 8;255(12):33-7.

[27].Labouta H I , Schneider M . Interaction of inorganic nanoparticles with the skin barrier: current status and critical review[J]. Nanomedicine: Nanotechnology, Biology and Medicine, 2013, 9(1):3954. 
[28].Baroli B , Ennas M G , Loffredo F , et al. Penetration of metallic nanoparticles in human fullthickness skin.[J]. Journal of Investigative Dermatology, 2007, 127(7):1701-1712.

[29]. Farjadian F , Ghasemi A, Gohari O, et al. Nanopharmaceuticals and nanomedicines currently on the market: Challenges and opportunities[J]. Nanomedicine, 2018(5).

[30]. Nelson, Durán, Camila, et al. Silver nanoparticle protein corona and toxicity: a mini-review.[J]. Journal of Nanobiotechnology, 2015.

[31]. Yuan X, Tay Y, Dou X, et al. Glutathione-protected silver nanoclusters as cysteine-selective fluorometric and colorimetric probe.[J]. Analytical Chemistry, 2013, 85(3):1913-1919.

[32]. Tang J , Xiong L, Wang S, et al. Distribution, translocation and accumulation of silver nanoparticles in rats.[J]. Journal of Nanoscience \& Nanotechnology, 2009, 9(8):4924.

[33]. Naumenko V, Nikitin A, Kapitanova K, et al. Intravital microscopy reveals a novel mechanism of nanoparticles excretion in kidney[J]. Journal of Controlled Release, 2019, 307.

[34]. Lankveld D P K, Oomen A G , Krystek P, et al. The kinetics of the tissue distribution of silver nanoparticles of different sizes[J]. Biomaterials, 2010, 31(32):8350-8361.

[35]. He X , Nie H, Wang K, et al. In Vivo Study of Biodistribution and Urinary Excretion of Surface-Modified Silica Nanoparticles[J]. Analytical Chemistry, 2008, 80(24):9597-9603.

[36]. Huang X, Li L, Liu T, et al. The shape effect of mesoporous silica nanoparticles on biodistribution, clearance, and biocompatibility in vivo.[J]. Acs Nano, 2011, 5(7):5390-5399.

[37]. Fu C , Liu T, Li L, et al. The absorption, distribution, excretion and toxicity of mesoporous silica nanoparticles in mice following different exposure route[J]. Biomaterials, 2013.

[38]. Patel B M , Paratz J, See N C, et al. Therapeutic drug monitoring of beta-lactam antibiotics in burns patients--a one-year prospective study.[J]. Therapeutic Drug Monitoring, 2012, 34(2):1604.

[39]. Fournier A, Eggimann P, Pantet O, et al. Impact of Real-Time Therapeutic Drug Monitoring on the Prescription of Antibiotics in Burn Patients Requiring Admission to the Intensive Care Unit[J]. Antimicrobial Agents \& Chemotherapy, 2017, 62(3):AAC.01818-17.

[40]. Li S D , Huang L . Pharmacokinetics and biodistribution of nanoparticles.[J]. Molecular Pharmaceutics, 2008, 5(4):496. 
[41]. Liu Y, Rohrs J, Wang P. Advances and challenges in the use of nanoparticles to optimize PK/PD interactions of combined anti-cancer therapies. Curr Drug Metab. 2014;15(8):818-28.

[42]. Ernsting MJ, Murakami M, Roy A, Li SD. Factors controlling the pharmacokinetics, biodistribution and intratumoral penetration of nanoparticles. J Control Release. 2013 Dec 28;172(3):782-94 .

[43]. Yang J , Chen Z, Lu D, et al. Glutathione capped silver nanoclusters-based fluorescent probe for highly sensitive detection of $\mathrm{Fe} \sim(3+)[\mathrm{J}]$. Sensors and Actuators, B. Chemical, 2014.

[44]. Hadrup N, Sharma AK, Loeschner K. Toxicity of silver ions, metallic silver, and silver nanoparticle materials after in vivo dermal and mucosal surface exposure: A review. Regul Toxicol Pharmacol. 2018 Oct;98:257-267.

[45]. Hadrup N, Lam HR. Oral toxicity of silver ions, silver nanoparticles and colloidal silver--a review. Regul Toxicol Pharmacol. 2014 Feb;68(1):1-7.

[46]. Alexander JW. History of the medical use of silver. Surg Infect (Larchmt). 2009 Jun;10(3):28992.

[47]. Beer C , Foldbjerg R , Hayashi Y, et al. Toxicity of silver nanoparticles - nanoparticle or silver ion?[J]. Toxicology Letters, 2012, 208(3):286-292.

[48]. Mijnendonckx K, Leys N, Mahillon J, Silver S, Van Houdt R. Antimicrobial silver: uses, toxicity and potential for resistance. Biometals. 2013 Aug;26(4):609-21.

[49]. Díez I, Ras RH. Fluorescent silver nanoclusters. Nanoscale. 2011 May;3(5):1963-70.

[50]. Norouzi M, Yasamineh S, Montazeri M, Dadashpour M, Sheervalilou R, Abasi M, PilehvarSoltanahmadi Y. Recent advances on nanomaterials-based fluorimetric approaches for microRNAs detection. Mater Sci Eng C Mater Biol Appl. 2019 Nov;104:110007.

[51]. Liu M, Tang F, Yang Z, Xu J, Yang X. Recent Progress on Gold-Nanocluster-Based Fluorescent Probe for Environmental Analysis and Biological Sensing. J Anal Methods Chem. 2019 Jan 2;2019:1095148. 
Figures
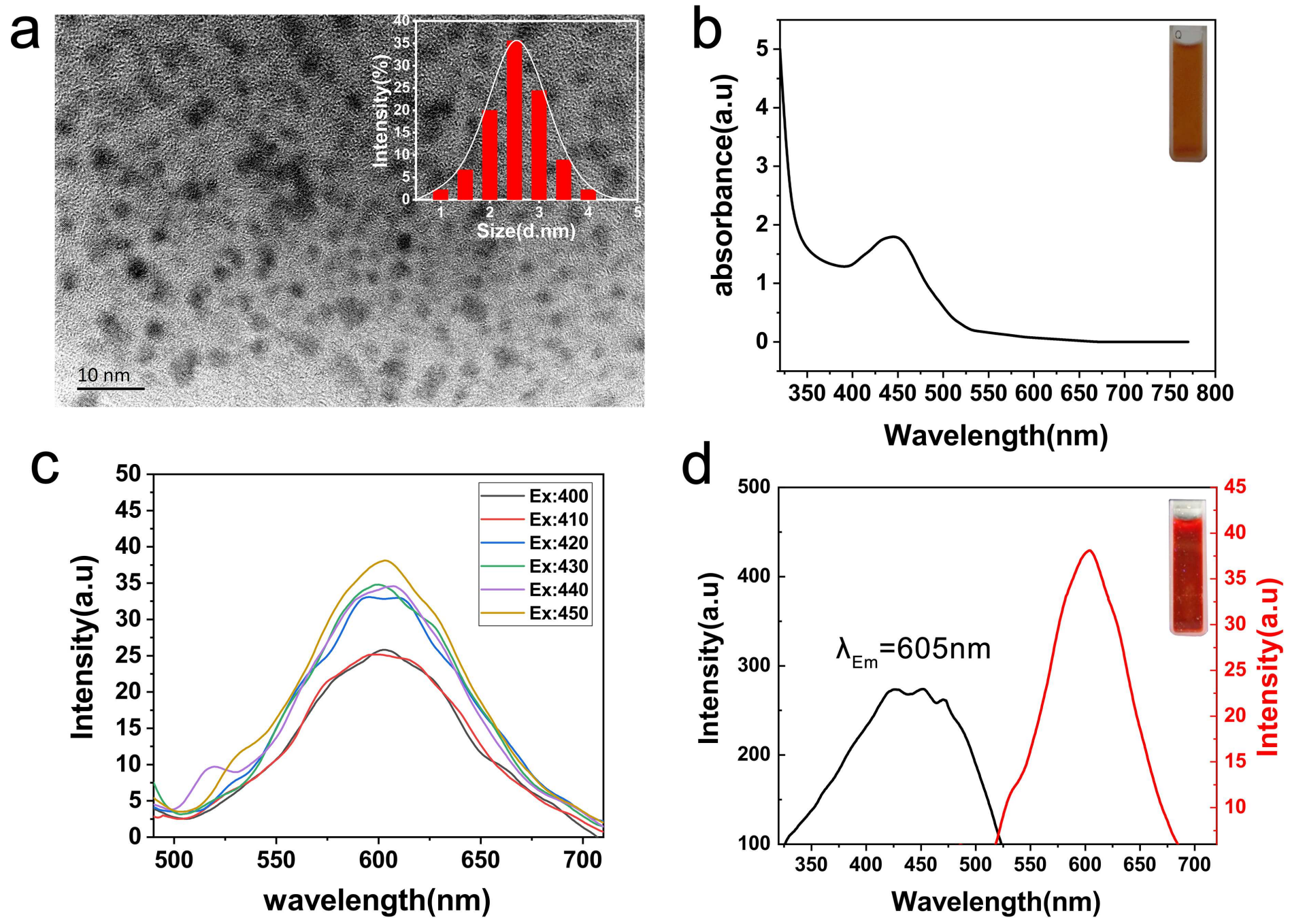

Figure 1

(a). TEM image of the GSH-AgNCs. Scale bar: $10 \mathrm{~nm}$. Illustration: Particle size distribution. (b). UV-vis spectroscopy of GSH-AgNCs, the inset shows the photo of GSH-AgNCs under visible light irradiation. (c). Changes in the fluorescence intensity of GSH-AgNCs measured under the action of different wavelengths of excitation light. (d). Excitation spectrum (black lines) and emission spectrum (red lines) of GSH-AgNC at $605 \mathrm{~nm}$ and $450 \mathrm{~nm}$. The inset shows a photo of GSH-AgNCs emitting red fluorescence under UV irradiation. 


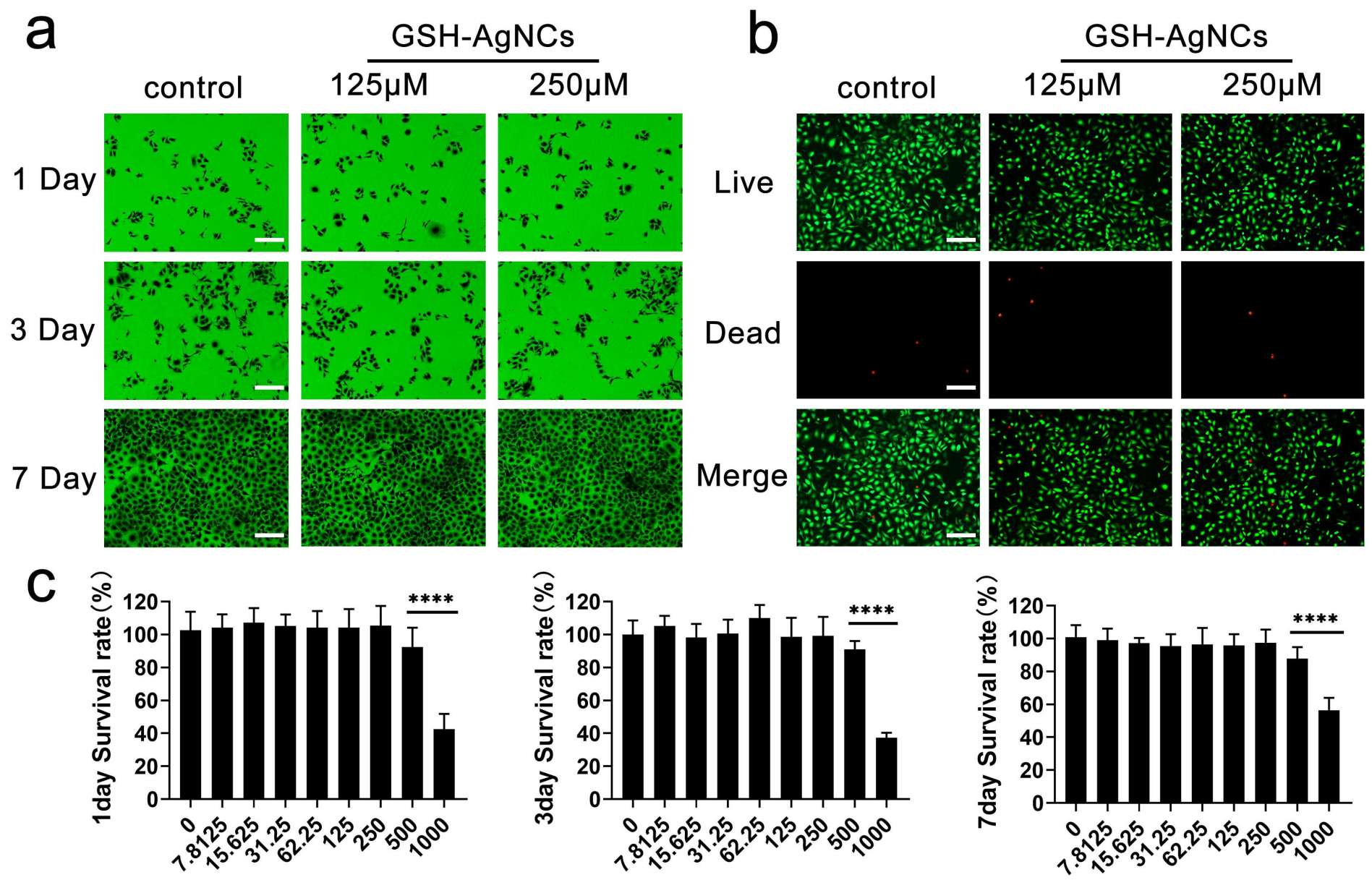

Figure 2

(a). Fluorescence inverted microscopy of the images of $125 \mu \mathrm{M}, 250 \mu \mathrm{M}$ GSH-AgNC, and HSF incubated together for 1, 3, and 7 days. Scale bar: $200 \mu \mathrm{m}$. (b). Live/dead fluorescent staining images of HSF cells incubated with different GSH-AgNCs after 5 days. Live cells are labeled with green fluorescence, while dead cells are labeled with red fluorescence. Scale bar: $200 \mu \mathrm{m}$. (c) The results of CCK-8 after co-culturing HSF cells with different concentrations of GSH-AgNCs for 1,3 , and 7 days. $\left({ }^{*} P<0.05\right)$. 

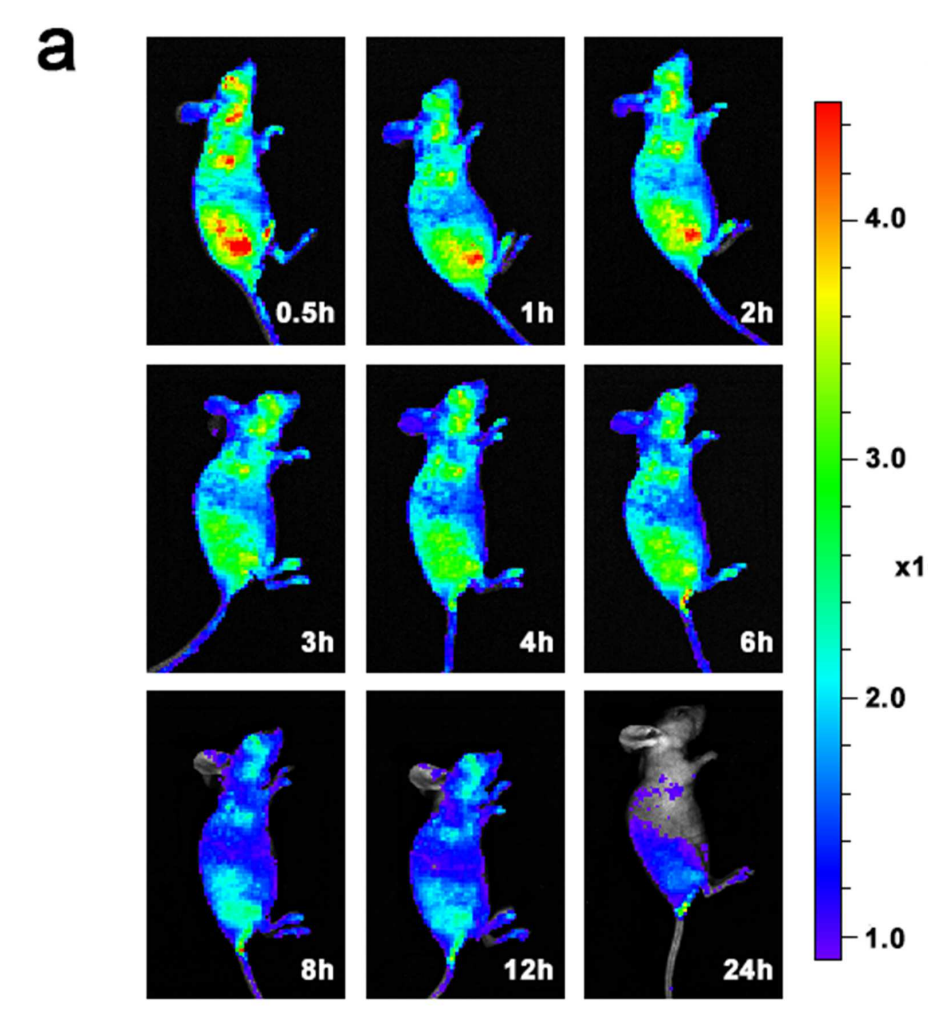

liver
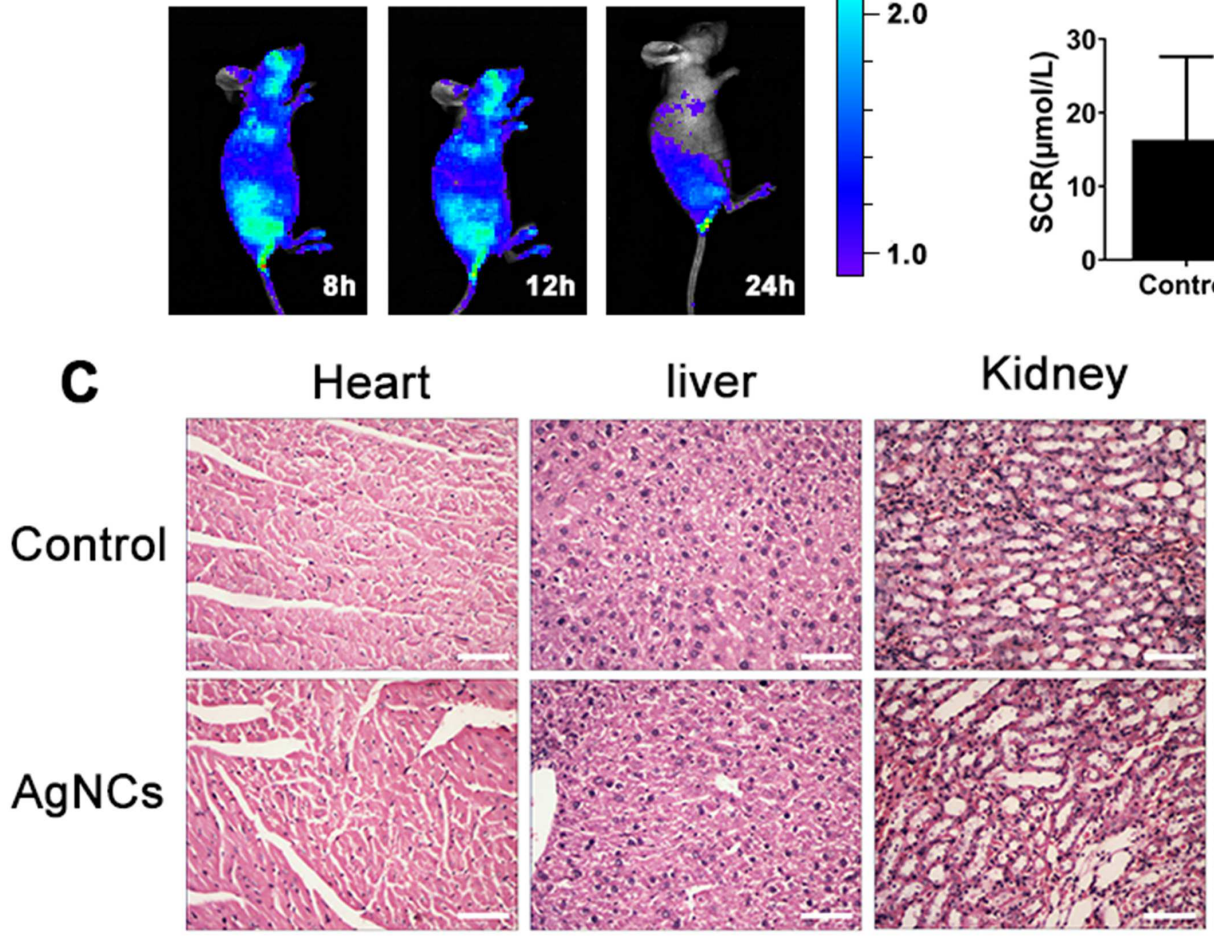
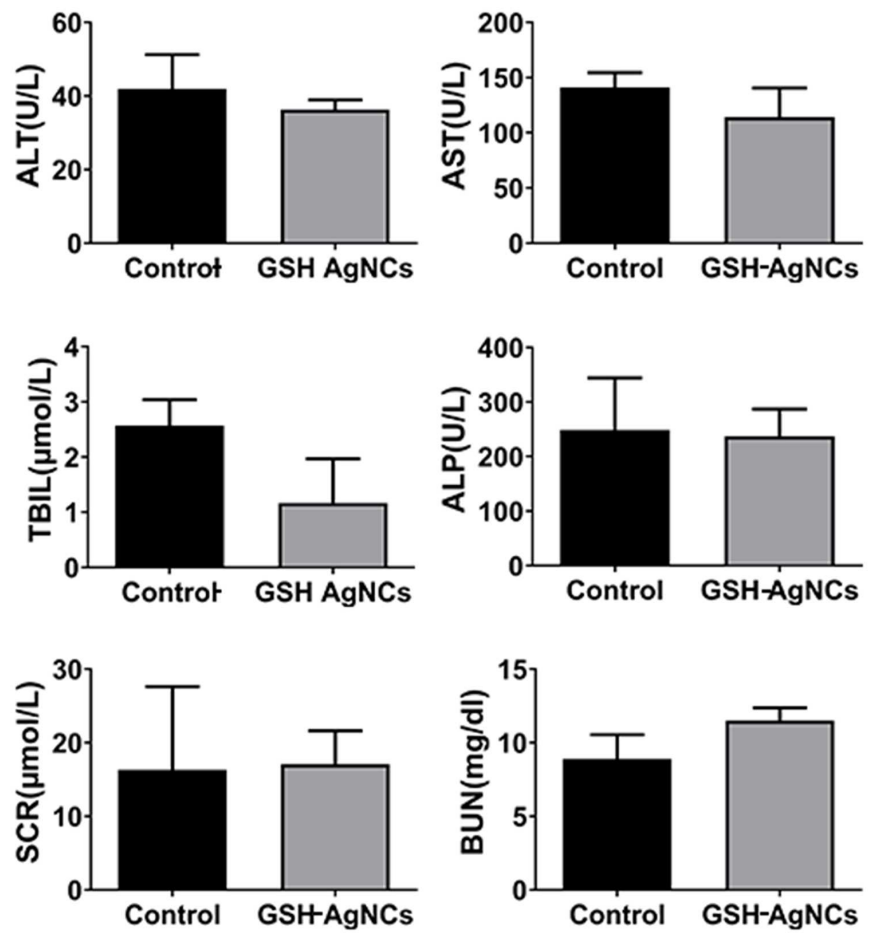

\section{Figure 3}

(a). Fluorescence images at different times point after injection of GSH-AgNCs. (b). Evaluation of the liver function (ALT, AST, TBIL, ALP)) and kidney function of km mice injected with GSH @ Ag NCs (BUN, SCR); mice in the control group received an intravenous injection of $200 \mu \mathrm{L}$ of normal saline. (c). Seven days after a single intravenous injection of GSH-Ag NCs suspension, H\&E-stained tissue (heart, liver, spleen, lung, and kidney) sections were taken from km mice. Scale bar: $200 \mu m$. $(n=3)$. 


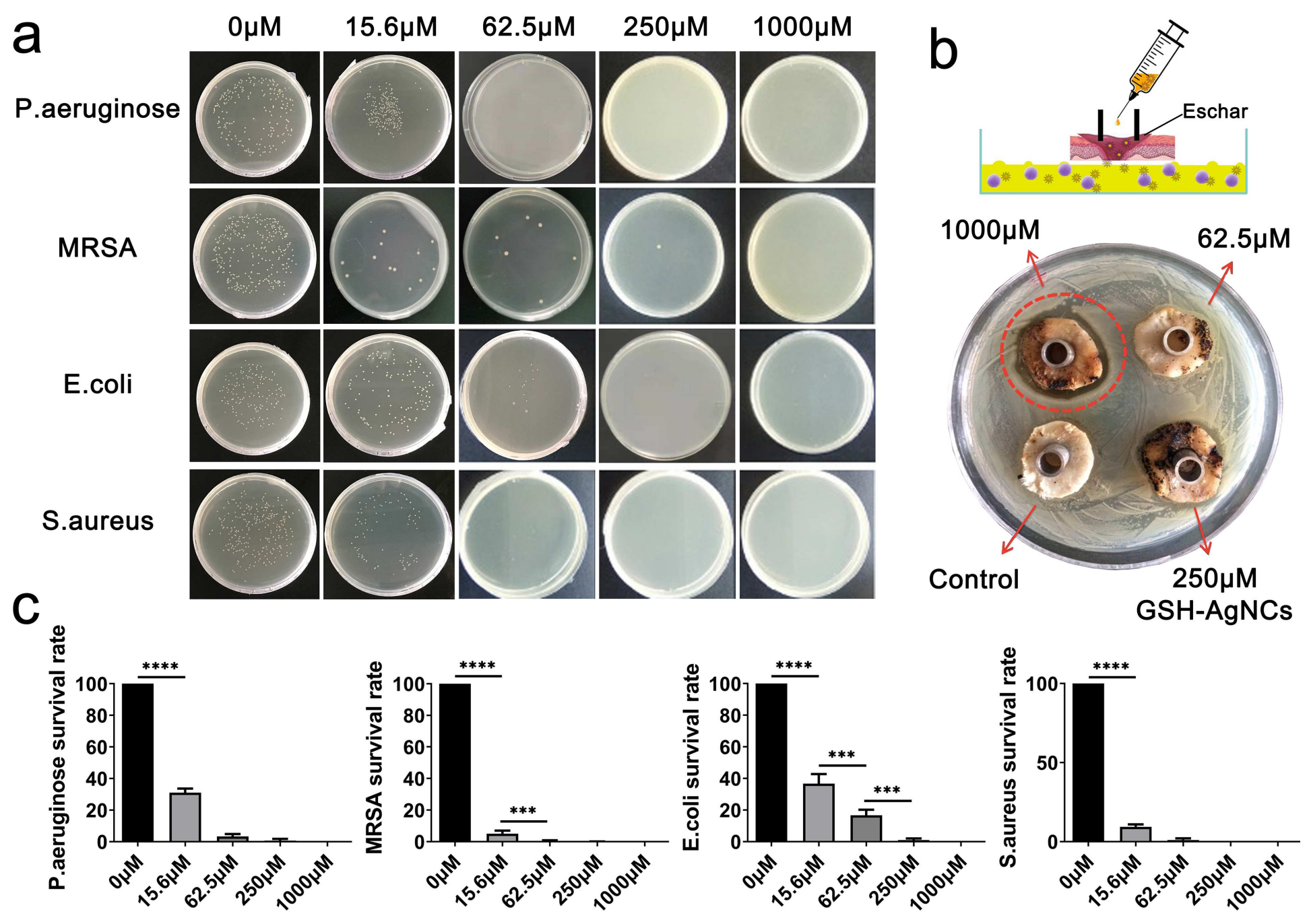

Figure 4

(a). Antibacterial efficiency of GSH-AgNCs against different bacteria using the Coated plate method. (b). The pictures and simple schematic diagrams of GSH-AgNCs permeating the skin eschar to form a zone of inhibition. (c). Histogram representation of the bacterial survival rate results. 
a

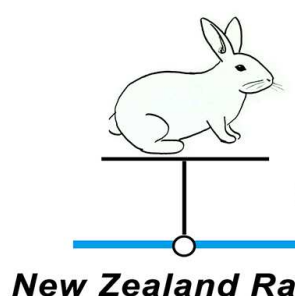

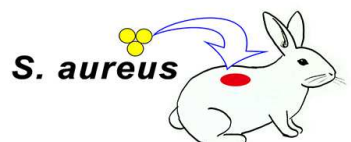

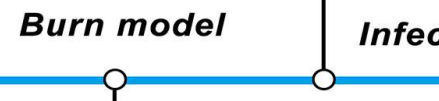

New Zealand Rabbit

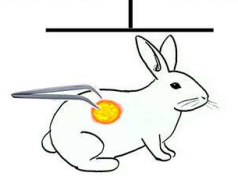

S. aureus Infection
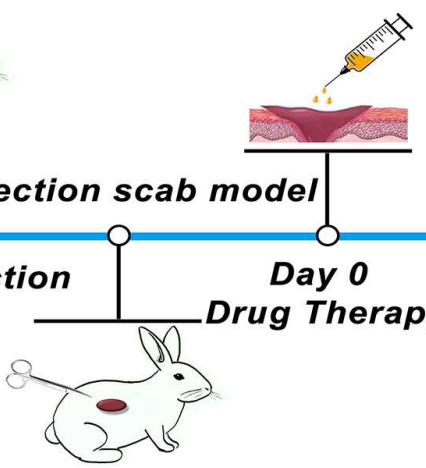

Day 0 Drug Therapy

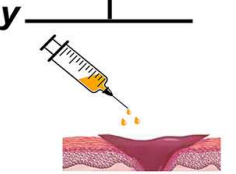

3day
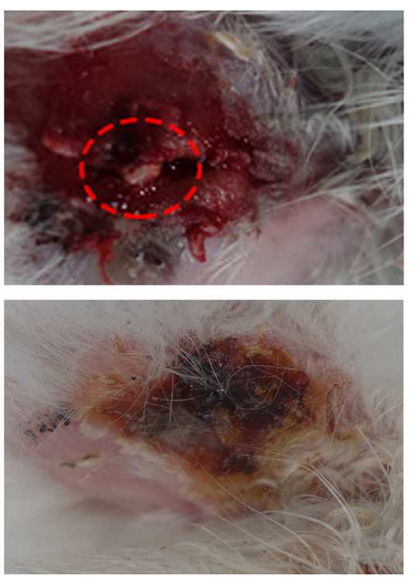

3day
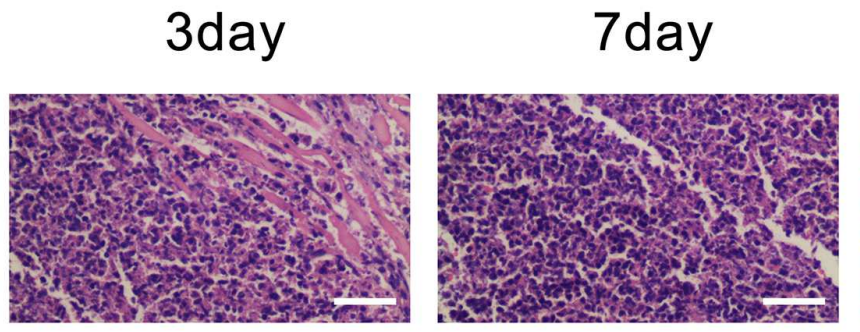

7 day

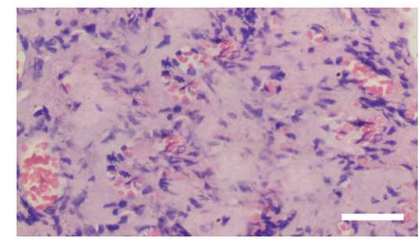

7 day
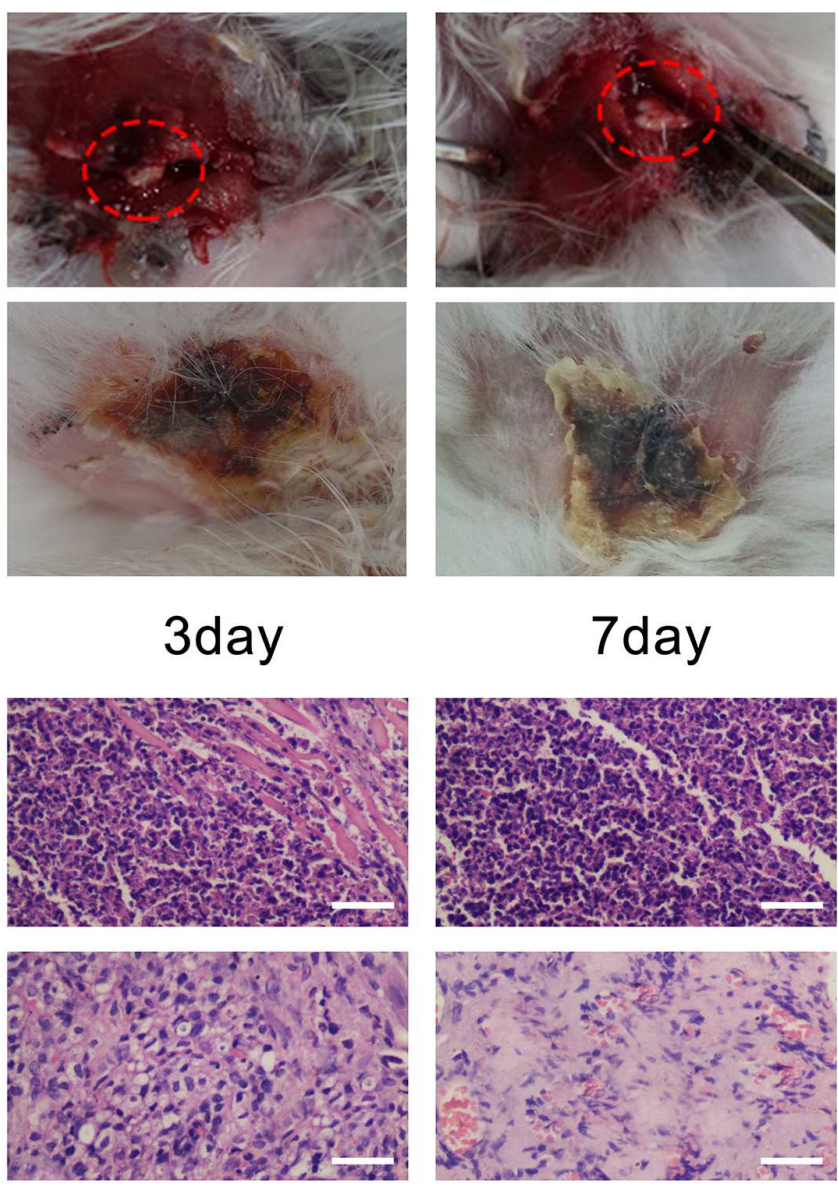
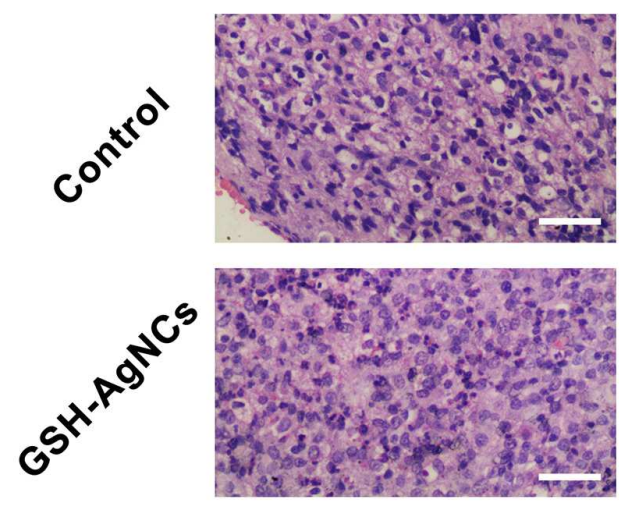

Figure 5

(a). Schematic diagram of the closed full-thickness burn wound infection modeling and GSH-AgNCs treatment process. (b). Wound healing efficacy of GSH-AgNCs in a burning infection wound model in rabbits; red circles indicate pus spots. (c). H\&E staining of the sampled wound tissues after 10 days posttreatment. Scale bar: $200 \mu \mathrm{m}$. 